\title{
Soft Electron Beam Lithography (Soft-eBL) Patterning of 0- and 1-D Nanostructures of Magnetic and Ferroelectric Oxides
}

Bin Liu*, and Vinayak P. Dravid*

*Department of Materials Science and Engineering, Northwestern University, Evanston, IL 60208, USA

Magnetic and ferroelectric oxide materials and their heterostructures possess a very broad spectrum of multifunctional properties related to electric, magnetic, stress field, and their combinations. The enhancement of these properties can result from the reduced size and the synergistic coupling between different component phases[1]. However, challenges still remain in the fabrication of uniformly textured ceramic nanopatterns and heterostructures that are spatially and dimensionally constrained, with multiple order parameters. Simultaneously, it is obviously important to obtain the underlying potentially novel structure-property relationships in the low dimensional systems with the aid of advanced and localized microscopy approaches.

We have fabricated 0 - and 1-D nanostructures of various spinel ferrite and perovskite oxides (and their composites) by soft-eBL, which combines variable pressure e-beam lithography with chemical solution deposition[2]. The attributes of variable pressure softeBL such as site-specificity, and high spatial resolution by reducing astigmatism and pattern distortion, are very well suited for heterostructure nanofabrication and microstructure control. By invoking the effects of single-crystal substrates with small lattice mismatch and heat-treatment profiles, oxide nanodisks (Fig. 1), nanolines (Fig. 2) and heterostructures are obtained.

Variable pressure scanning electron microscope (VP-SEM) and atomic force microscope (AFM) were used to characterize the faceted morphology of the various oxide nanostructures. Plan-view and cross-sectional TEM samples were prepared by traditional methods and focused ion beam (FIB). Electron diffraction and high resolution TEM (HRTEM) indicated the epitaxial growth and single crystallization of the oxides. HRTEM also revealed the interfaces and related strains between the substrates and the deposited materials, or between different phases in the heterostructures, which have been shown to be very important for the piezoelectric response and the coupling in the multiferroic heterostructures[3]. The magnetic and ferroelectric properties were studied by SQUID, magnetic force microscopy and piezoelectric force microscopy. The presentation will cover synergy, complementarities and advantages of combined SPM/S/TEM and FIB approaches to analysis of nanostructure under spatial and dimensional constraints.

\section{References}

[1] W. Eerenstein, N. D. Mathur and J. F. Scott, Nature 442 (2006) 759. 
[2] Z. Pan et al., Nano Letters 6 (2006) 2344.

[3] W. Lee et al., Nat. Nanotechnol. 3 (2008) 402.

[4] Z. Pan et al., Applied Physics Letters 91 (2007) 143105.

[5] This work was supported by the NSF-MRSEC, and the U.S. Department of Energy (DOE-BES) programs. The research work was performed in the EPIC and NIFTI facilities of the NUANCE Center at Northwestern University. NUANCE Center is supported by NSF-NSEC, NSF-MRSEC, the State of Illinois, and Northwestern University.

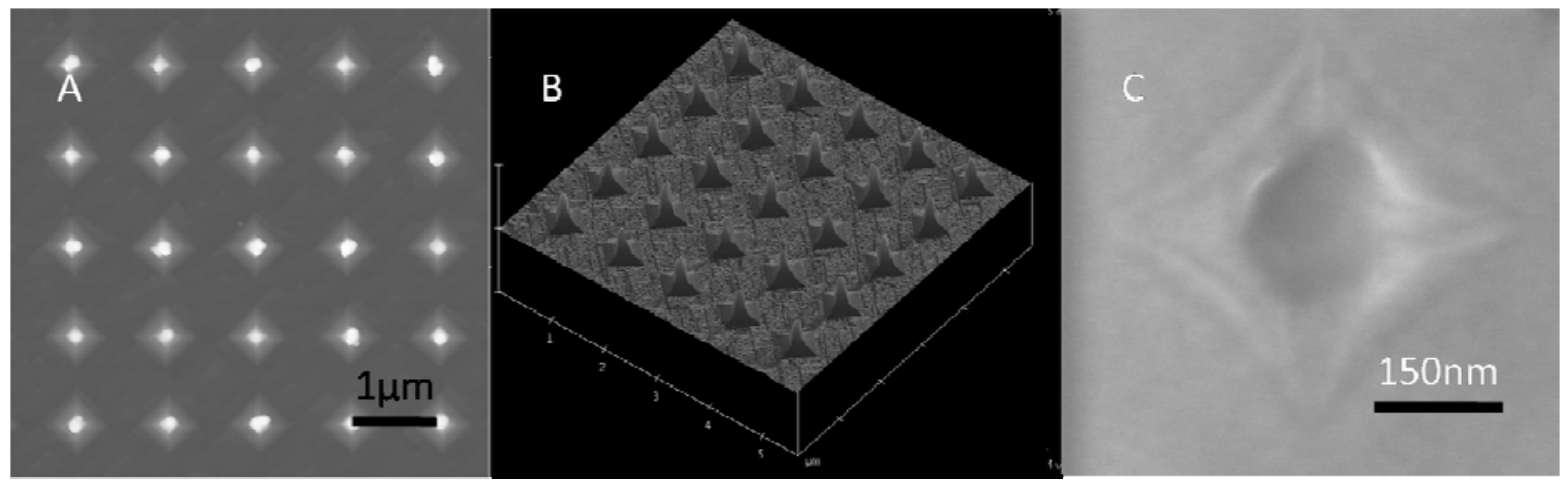

Fig. 1 Cobalt ferrite nanodisks on $\mathrm{MgO}$ substrate. $\mathrm{A}$ and $\mathrm{B}$ are $\mathrm{AFM}$ height image and 3D image. C is VP-SEM image.

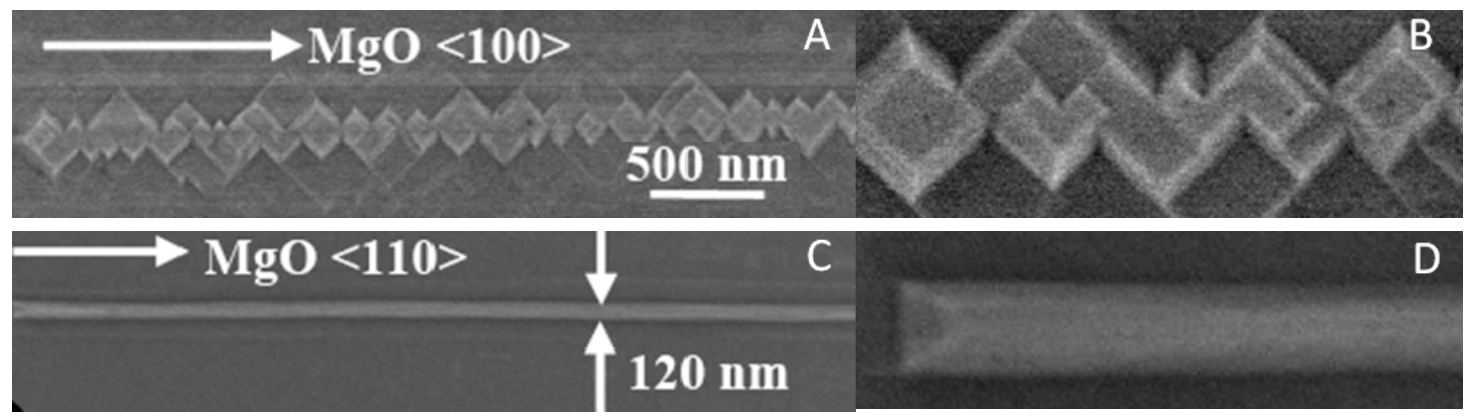

Fig. 2 Cobalt ferrites nanolines on $\mathrm{MgO}$ substrates[4]. A and B are VP-SEM images of $\mathrm{CFO}$ lines patterned along $\langle 100\rangle_{\mathrm{MgO}}$ directions. $\mathrm{C}$ and $\mathrm{D}$ are VP-SEM images of CFO lines patterned along $\langle 110\rangle_{\mathrm{MgO}}$ direction. 